\title{
The long-term effects of pre-treatment with activated protein $C$ in a rat model of compression-induced spinal cord injury
}

\author{
Y Taoka ${ }^{1,2}$, MG Schlag*,3, R Hopf ${ }^{1}$ and H Red1 ${ }^{1}$ \\ ${ }^{1}$ Ludwig Boltzmann Institute for Experimental and Clinical Traumatology, Donaueschingenstrasse 13, A-1200 Vienna, \\ Austria; ${ }^{2}$ Department of Orthopedic Surgery, School of Medicine, the University of Tokushima, 3-18-15 Kuramoto- \\ cho, Tokushima, 770-8503, Japan; ${ }^{3}$ Baxter AG, Industriestr. 20, A-1221, Vienna, Austria
}

\begin{abstract}
Objectives: Recently, we demonstrated that activated protein C (APC) can lessen the severity of spinal cord injury (SCI) in rats during the acute and subacute phases. The purpose of the present study is to determine the long-term effects of pre-treatment with APC following SCI in rats.

Methods: The motor function of rats was assessed using the inclined-plane test during 8 weeks after SCI, and the grid runway test 7 weeks after the trauma. Somatosensory evoked potentials (SEPs), brainstem-derived motor evoked potentials (B-MEPs) and corticomotor evoked potentials (CMEPs) were used to quantify axonal function 8 weeks after SCI. Morphometric analysis of the spinal cord lesion was carried out to determine lesion size. Twelve male Sprague-Dawley rats were randomly allocated to either APC (25 IU/ $\mathrm{kg}$ ) or saline group and then subjected to $20 \mathrm{~g}$ compression injury of the spinal cord for $20 \mathrm{~min}$ at T12. The sham group $(n=6)$ received laminectomy alone.

Results: APC significantly reduced the motor disturbances and electrophysiological impairments induced by SCI. APC-treated animals also showed a trend towards a reduction in lesion size. However, this change, was not significant.

Conclusion: Pre-treatment with APC attenuates the harmful effects of SCI not only during the acute and subacute phases but also in the chronic stage.
\end{abstract}

Spinal Cord (2000) 38, 754-761

Keywords: spinal cord injury; activated protein C; motor disturbance; evoked potentials; lesion size

\section{Introduction}

Acute traumatic spinal cord injury (SCI) is an unexpected, catastrophic event, the consequences of which often persist for the life of the patient and influence in diverse ways not only the patient, but also family members and society at large. ${ }^{1}$ Only limited therapeutic measures are currently available for its treatment. $^{2}$ Many experimental studies have demonstrated that pathophysiologic changes after acute SCI are consistent with a two-step mechanism. The primary injury is caused by an external force and mainly represents mechanical tissue damage. This initial injury sets off a cascade of secondary autodestructive processes which cause further tissue loss. ${ }^{3,4}$ The latter processes can be attenuated by therapeutic intervention. Although the mechanisms leading to this progressive damage are not fully understood, endothelial damage and leukocyte infiltration have been shown to play an important role during secondary tissue destruction. ${ }^{5,6}$

*Correspondence: MG Schlag, Baxter AG, Industriestr. 20, A-1221, Vienna, Austria
Activated protein C (APC) is an important physiological anticoagulant that is generated from protein $\mathrm{C}$ by the action of the thrombin-thrombomodulin complex on the endothelial cells. ${ }^{7}$ APC inactivates factors $\mathrm{Va}$ and VIIIa, thereby regulating the coagulation system. ${ }^{7,8}$ APC is also implicated in the regulation of inflammatory processes by its inhibition of cytokine production by monocytes. ${ }^{9}$ Recently, we demonstrated that APC prevented the secondary effects of trauma-induced SCI at acute and subacute phases by inhibiting neutrophil activation. ${ }^{10}$ However, the long-term effects of APC on SCI are not well documented. In the present study, we investigated the effects of APC over an extended period of time after traumatic SCI in rats.

\section{Methods}

Surgical interventions

Male Sprague-Dawley rats, weighing 300-350 g, were obtained from the Institut fuer Labortierkunde und- 
genetik der Medizinischen Fakultaet der Universitaet Wien (Himberg, Austria). APC was kindly provided by Baxter (Vienna, Austria). ${ }^{11}$ All other reagents used were of analytical grade.

The study protocol was approved by the Animal Care and Use Committee of the City of Vienna. Male Sprague-Dawley rats were anesthetized with intraperitoneal (i.p.) injections of ketamine $(75 \mathrm{mg} / \mathrm{kg})$, xylazine $(10 \mathrm{mg} / \mathrm{kg})$, and atropine $(0.3 \mathrm{mg} / \mathrm{kg})$. For maintenance of anesthesia, half of the initial ketamine/ xylazine/atropine dose was administered i.p. every $60 \mathrm{~min}$. Rectal temperature was maintained at $37-$ $38^{\circ} \mathrm{C}$ with a heating lamp until recovery.

Rats were randomly assigned to one of three groups (sham, APC, control) prior to surgery. Each rat was subjected to laminectomy at the level of the 12th thoracic vertebra (T12). In 12 rats, spinal cord injury (SCI) was induced by applying a 20-g weight extradurally to the spinal cord at T12 for $20 \mathrm{~min}$ as previously described. ${ }^{6,10}$ This technique causes paresis of the lower extremities in a reproducible manner. ${ }^{6,12}$ Six rats received intravenous APC (25 IU/kg, $100 \mathrm{IU} /$ $\mathrm{ml}$; Baxter, Vienna) $30 \mathrm{~min}$ before the compression trauma. The control animals (control; $n=6$ ) received saline instead of APC. Laminectomy alone was performed as sham operation (sham; $n=6$ ). Before recovery, all rats received subcutaneous injections of $10 \mathrm{ml}$ Ringer's solution and antibiotics (50 $000 \mathrm{IU}$ Procaine-penicillin-G, 50000 IU Dihydro-streptomycinsulfate; Omnamycin, Hoechst). The animals were housed under controlled light and temperature conditions and were fed standard rat chow and water. They were checked daily for signs of infection or dehydration. Manual bladder expression was carried out until spontaneous voiding returned.

\section{Grading of motor disturbance}

The motor function of rats was assessed using the inclined-plane test, ${ }^{10,13}$ and the grid runway test. ${ }^{14}$ In the inclined-plane test, recovery from motor disturbance was assessed before, and again at 1, 7, 14, 21, 28, $35,42,49$, and 56 days after the compression. We recorded the maximum inclination of the plane on which the rats could maintain themselves for $5 \mathrm{~s}$ without falling. ${ }^{10}$

In the grid runway test, rats were trained in the 6th post-operative (p.o.) week and tested in the following week ( 7 th p.o. week). Water was removed on the first day of training and was kept withdrawn for the entire training and testing period. After the daily training and testing sessions the rats were allowed free access to water for $30 \mathrm{~min}$. Animals' weights were measured daily and supplemental fluid (Ringer's solution) was given if progressive weight loss was noted $(60 \mathrm{ml} / \mathrm{kg}$ day). All animals reached a stable weight 3-4 days after water withdrawal. The rats were trained to cross a grid runway $(20 \times 200 \mathrm{~cm} ; 2 \mathrm{~mm}$ wire diameter, $30 \times 30 \mathrm{~mm}$ grid holes) for a water reward presented in a water dispenser. Training was daily for 5 days (four crossings/day). Following the training period the rats were tested for 5 consecutive days. The animals were observed while crossing the runway (four crossings/ day) for a water reward presented in a water dispenser. The number of footfalls of each hind limb per crossing was counted and recorded. An average of all values was calculated to obtain a single value for each rat.

\section{Electrophysiological measurements}

At the end of the survival period (8 weeks), electrophysiological measurements were done according to a protocol previously described. ${ }^{15,16}$ The measurements were done by an investigator unaware of group assignment (MG Schlag). Each rat had three stainless steel screws $(4 \mathrm{~mm}$ length, $1.3 \mathrm{~mm} \varnothing)$ implanted extradurally into the skull. Two screws were positioned over the hind limb areas of the left and the right sensorimotor cortex (coronal screws) at following coordinates: $2.5 \mathrm{~mm}$ lateral to midline and $3 \mathrm{~mm}$ posterior to bregma. The third screw (occipital screw) was positioned $1-2 \mathrm{~mm}$ posterior to the lambda, thus overlying the transverse fissure. The coronal and occipital screws were inserted 1 and $1.5 \mathrm{~mm}$ deep, respectively. Stimulation was carried out with a Neuromax (Excel Tech, Toronto, Canada). During stimulation, a ground needle electrode was placed subdermally over the dorsum of the neck. Signals were filtered at a bandpass frequency between 10 and $3000 \mathrm{~Hz}$ and stored on a computer for later analysis.

For somatosensory evoked potentials (SEPs) recording, the hind paw was stimulated via surface electrodes positioned around the ankle (cathode) and the middle of the paw (anode). Stimuli consisted of $100 \mu \mathrm{s}$ rectangular biphasic impulses delivered at $2.7 \mathrm{~Hz}$. At least two signals were recorded at supramaximal stimulation intensities $(2-3 \mathrm{~mA})$ from the contralateral coronal screw (anode) referenced to the posterior screw (cathode), with sweep length set to $60 \mathrm{~ms}$. Each signal represents the average of 60 repetitions. In animals showing no or reduced SEPs, stimulation intensities were doubled (4-6 mA).

For brainstem-derived motor evoked potentials (BMEPs), single $100 \mu$ s rectangular biphasic impulses were applied via the posterior screw (cathode) with a needle electrode in the submucosa of the palate (anode). Signals were recorded at supramaximal stimulation intensities $(10-20 \mathrm{~mA})$ from both hind limbs.

Corticomotor evoked potentials (CMEPs) were elicited by repeated, high-frequency bipolar stimulation. Trains of low intensity impulses (100 $\mu \mathrm{s} ; 5-$ $12 \mathrm{~mA}$; $15.1 \mathrm{~Hz}$ ) were applied via one cortical screw (anode) with the cathode attached to the posterior screw. In order to avoid co-stimulation of other motor areas, the trains were kept short $(10-20$ impulses $)$ and stimulation was stopped when contraction of the contralateral hind limb was noted. We have previously shown that this method yields signals that are conducted by the corticospinal tract: transection of the 
corticospinal tract completely and irreversibly abolished the signals. ${ }^{16}$ Thus, these signals can be considered to be of true cortical origin. Signals were recorded from the tibialis anterior muscle of the contralateral hind limb. In case no signal could be elicited, numbers of repetitions and/or stimulation intensities were increased until front limb muscle contraction and movements of the vibrissae were noted. This was done to ensure that stimulation intensities would have been sufficient to stimulate CMEPs in an animal with an intact spinal cord.

B-MEPs and CMEPs were both recorded via monopolar needles inserted into the belly of the tibialis anterior muscles, with the reference electrode inserted into the footpad. Sweep length was 24 and $60 \mathrm{~ms}$ for B-MEPs and CMEPs, respectively. At least two signals were recorded to ensure reproducibility.

The recorded potentials were analyzed by measuring latency and amplitudes. Latency was defined as the time between the onset of the stimulus artifact to the first positive or negative peak. Amplitudes were measured from baseline to first peak (SEP, B-MEP: N1) and between peaks for the consecutive peaks (SEP: P1, N2), or represented the largest distance between two consecutive peaks of a polyphasic signal (CMEP). Because SEPs consist of three consecutive peaks in healthy animals, but are often reduced to a single peak after injury, the amplitudes of the individual peaks were summed to obtain a single value. The values obtained for left and right hind limbs were averaged.

\section{Histological examination of the spinal cord}

After completion of the electrophysiological recordings, the rats were transcardially perfused under deep anesthesia with $50 \mathrm{ml}$ of Ringer's solution followed by $50 \mathrm{ml}$ of $10 \%$ phosphate buffered formalin $(\mathrm{pH}$ 7.4). The upper lumbar spinal cord was dissected out and postfixed in the same solution for at least $24 \mathrm{~h}$. A segment of the spinal cord containing the lesion plus $2 \mathrm{~mm}$ rostral and caudal to the lesion was embedded in paraffin. Serial sections $(5 \mu \mathrm{m})$ were taken at $100-\mu \mathrm{m}$ intervals. One set of serial sections was stained with hematoxylin and eosin in order to study gray matter destruction, while the second set was stained with Luxol blue in order to determine the extent of the remaining functional white matter. In the sham group, single histological sections of the spinal segment L2 were obtained. Sections were viewed with a microscope fitted with a digital camera (Coolpix950; Nikon). Digital images of sections showing pathological changes or lesions of the gray matter were analyzed with morphometric analysis software (Lucia G, Laboratory Imaging, CZ). The following parameters were determined by an investigator unaware of group assignment (MG Schlag).

Spared white matter The section representing the largest extent of the lesion was determined and the area of the spared white matter measured (Figure 1). This value was expressed as percentage of the average cross-sectional area of an undamaged spinal cord (sham group).

Length of gray matter lesion Lesion borders were identified by one or several of the following markers: (i) gray matter cavities; (ii) absence of neurons with normal appearance and (iii) presence of reactive glia (Figure 2). The length of the gray matter lesion was calculated by multiplying the number of sections presenting gray matter injury with 120 (actual length between sections, including $100 \mu \mathrm{m}$ interval and four sections at $5 \mu \mathrm{m}$ ) and a shrinking factor which was calculated from in situ length, post-fixation length and post-embedding length.

Summed area of gray matter lesion Gray matter loss was expressed as the total area of the gray matter lesion. Starting at one end of the lesion and stopping at the other end, the remaining gray matter was measured (Figure 2). In case no gray matter was discernible in a given section, the value was set to zero. These values were then subtracted from the gray matter measured in the first rostal or caudal sections without gray matter injury (depending on which section was closest). Thus, an estimate of the area of gray matter lesion in a given section was obtained. These values were then summed to obtain a single value for each animal. To compensate for inter-animal differences regarding the area of unlesioned gray matter, an average was calculated from all values of unlesioned gray matter. The individual values of unlesioned gray matter were then expressed as a fraction of this average and the summed values of gray matter lesion area multiplied with this fraction.

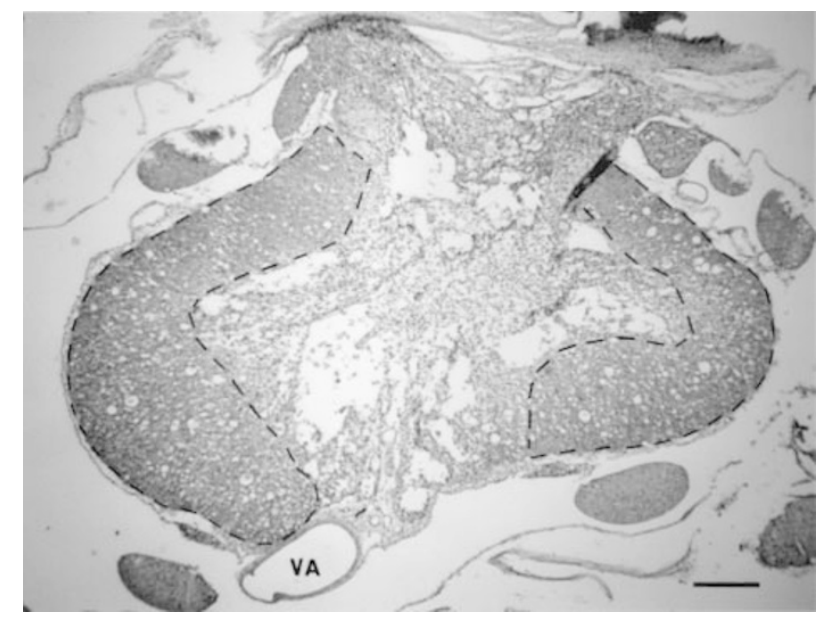

Figure 1 Micrograph of a luxol blue stained cross-section of a lesioned spinal cord. The section represents the maximal extent of the spinal cord lesion in an untreated control animal 8 weeks after SCI. A big central cyst has formed. The area of surviving white matter is outlined (- - ). VA, ventral artery; bar $=200 \mu \mathrm{m}$ 


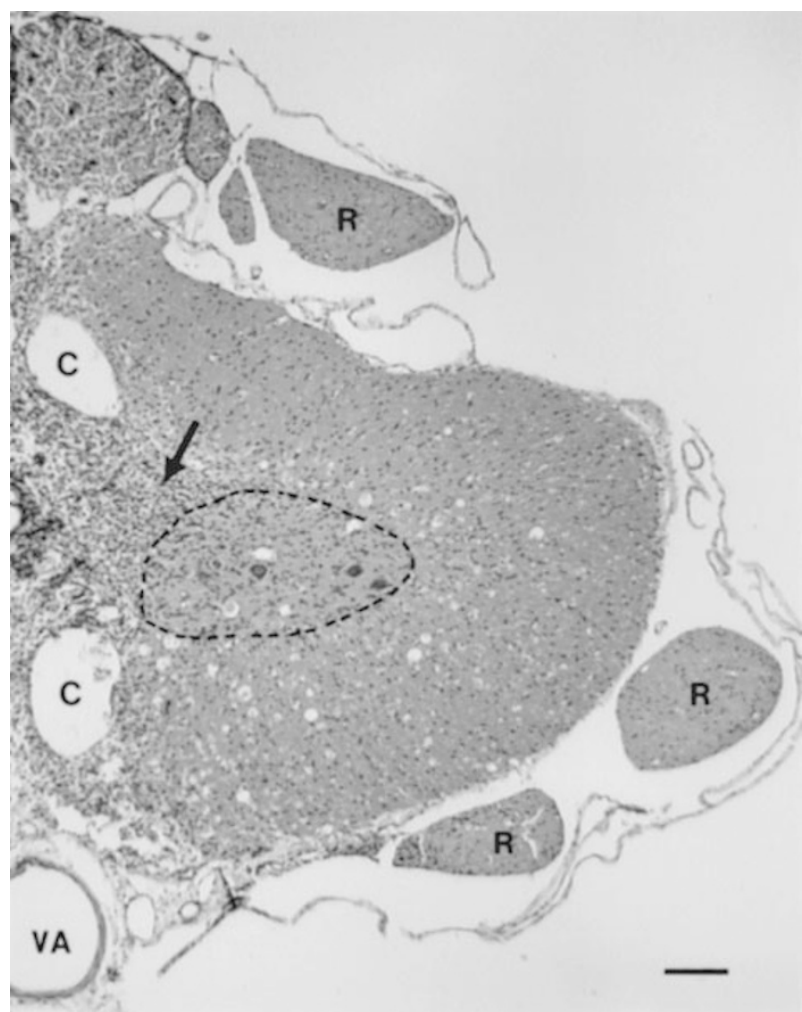

Figure 2 Micrograph of a $\mathrm{H}$-E stained cross-section of a lesioned spinal cord. The section was taken from the tapered end of the spinal cord lesion in an APC-treated animal 8 weeks after SCI. The surviving gray matter is outlined (- - ). The arrow points to an area of extensive gliosis. C, cyst; R, spinal nerve root; VA, ventral artery; bar $=100 \mu \mathrm{m}$

\section{Statistical analysis}

All data, except the angle of the inclined plane, were analyzed by one-way Analysis of Variance (ANOVA) with group as factor. In case a major deviation from the normality of the inter-animal data was observed, an Analysis of Variance on the ranks (Kruskal-Wallis test) was used. Because the data of the inclined plane test showed a non-parametric distribution, an Analysis of Variance on the ranks (Kruskal-Wallis test) was performed. If ANOVA detected a significant difference, post hoc analysis was carried out (Student-NewmanKeuls-test). In case multiple comparisons were done, a Bonferoni correction factor was calculated.

\section{Results}

\section{Motor function}

When evaluated by the inclined plane test, we found that APC-treatment remarkably reduced the motor disturbances induced by compression trauma during the total 8-week observation period.

Immediately following compression, the average maximum angles in both the untreated control and the APC-treatment groups were significantly smaller than in the sham group. In the untreated control group, the average maximum angle remained below sham values throughout the 8 -week observation period despite a marked recovery during the first 21 postoperative (p.o.) days and a second slight improvement of scores after p.o. day 42 (Figure 3). Significant differences between the sham and the untreated control groups were found on p.o. days 1, 7, 35, and 42. In contrast, in the APC-treated group, the average maximum angle reached sham levels on p.o. day 7 , and no significant difference between the APCtreatment and sham groups was found during the remaining survival period (Figure 3).

APC-treatment also improved motor function when evaluated by the grid test. Number of foot falls/ crossing in the untreated control group was significantly higher than in the sham group. APC-treatment reduced the number of foot falls/crossing down to sham levels (Figure 4).

\section{Electrophysiology}

SEP In the sham group, SEPs consisted of three consecutive peaks (N1: $15.5 \pm 0.8 \mathrm{~ms} ; \mathrm{P} 1: 20.4 \pm 1 \mathrm{~ms}$; N2: $27.9 \pm 1.3 \mathrm{~ms}$; Figure $5 \mathrm{~A}$ ). In the untreated control, a single broad negative peak with significantly longer latency replaced the tri-phasic signal (N1: $20.3 \pm 1.7 \mathrm{~ms}$; Figures 5B and 6). APC-treated animals showed typical SEPs with three consecutive peaks whose latencies did not differ significantly from sham values $(\mathrm{N} 1: \quad 17.7 \pm 2.9 \mathrm{~ms} ; \quad \mathrm{P} 1: \quad 20.8 \pm 1.9 \mathrm{~ms} ; \quad \mathrm{N} 2$ $28.4 \pm 2 \mathrm{~ms}$; Figures $5 \mathrm{C}$ and 6 ). Trauma not only changed SEP configuration and latencies but also significantly reduced summed peak amplitudes (Figure

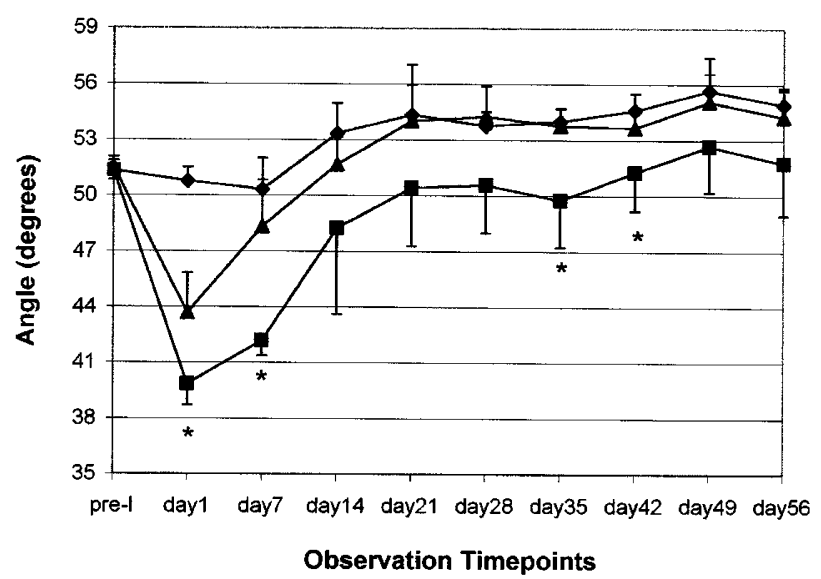

Figure 3 Temporal effect of APC on motor disturbances after compressive SCI as determined by the inclined plane test. APC-treatment remarkably reduced the motor disturbances induced by compression trauma during 8 weeks after SCI. Closed square, untreated control animals; closed triangles, APC-treated animals; closed diamond, shamoperated animals. Mean $\pm \mathrm{SD}$ of six experiments. ${ }^{*} P<0.05$ versus APC-treated and sham animals 
7A). By contrast, summed SEP amplitudes in the APCtreated animals did not differ from sham values.

$B$-MEP B-MEP consisted of a prominent negative peak (N1, latency: $5.5-7.2 \mathrm{~ms}$ ) frequently followed by 1 to 4 peaks of variable latencies. While compression did not affect N1 latency (sham: 6.7 $\pm 0.4 \mathrm{~ms}$, untreated control: $6.7 \pm 0.2 \mathrm{~ms}$, APC: $6.6 \pm 0.5 \mathrm{~ms}), \mathrm{N} 1$ amplitude was significantly depressed in the untreated control group, whereas no difference was found between sham and APC-treated groups (Figure 7B).

CMEP Stimulation of the cortex resulted in longlatency polyphasic signals $(10-20 \mathrm{~ms}$ duration; Figure $8 \mathrm{~A})$ that were accompanied by distinct contractions of the contralateral hind limb. The initial peak had variable polarity and its latency ranged between $15-$ $21 \mathrm{~ms}$. CMEPs were abolished after compression (Figure 8B) but were preserved by APC treatment (Figure 8C). CMEP latencies (sham: $19.3 \pm 1.7 \mathrm{~ms}$; APC: $19.3 \pm 1.9 \mathrm{~ms}$ ) and amplitudes (Figure 7C) were similar in sham and APC-treated groups.

\section{Histology}

All three parameters indicated a smaller lesion size in the APC-treatment group compared to the untreated control group (Table 1). However, these differences did not reach significance. Of all three parameters, the summed area of gray matter lesion showed the largest difference between APC-treatment and untreated control group $(P=0.069)$.

\section{Discussion}

In a previous study, we demonstrated that APC could lessen the severity of SCI. However, observations of the APC-treated animals in that study were restricted to the acute and subacute recovery phases. ${ }^{10}$ In the present study, we wanted to elucidate the long-term effects of APC-treatment on SCI and determine the

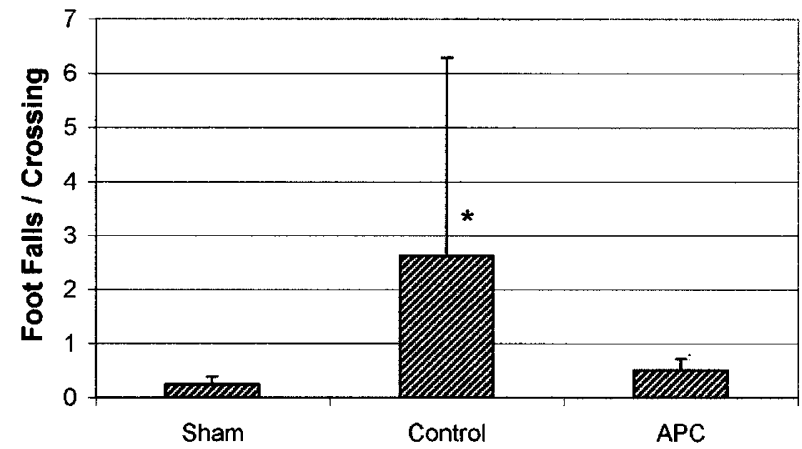

Figure 4 Effect of APC on motor disturbances 7 weeks after compressive SCI as determined by a grid test. APC-treatment reduced the motor disturbances induced by compression trauma. Mean \pm SD of six experiments. ${ }^{*} P<0.05$ versus sham animals
A

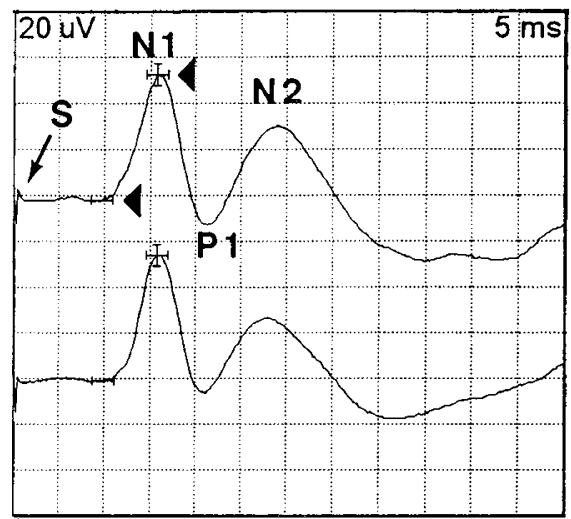

B
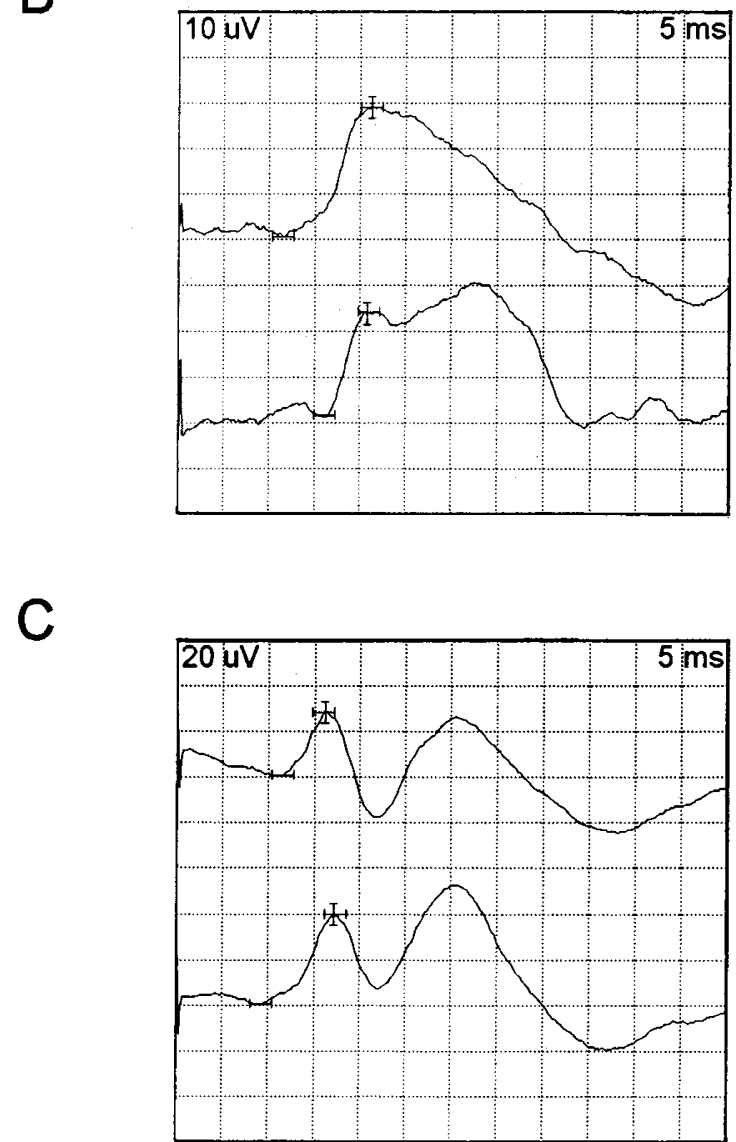

Figure 5 Effect of APC on SEPs. SEPs in the untreated control group (B) had a markedly different configuration than SEPs in both the sham (A) and the APC-treated groups (C). Representative recordings are shown. (A) Upper signal; s, stimulus artifact; N1, first negative deflection; P1, first positive deflection; N2, second negative deflection; the vertical cursor marks N1 peak latency, the horizontal cursors (arrowheads) indicate N1 amplitude. Note that in this and all subsequent recordings upward deflections are negative 
neurological and histological correlates of functional recovery. We found that SCI-induced motor distur-

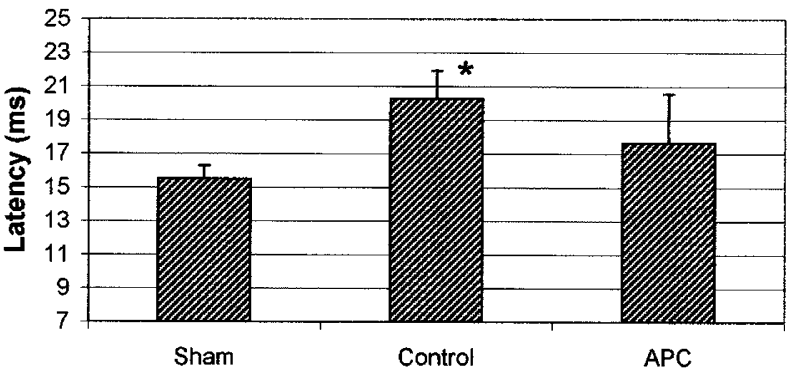

Figure 6 Effect of APC on the latency of SEPs. SEPs latencies were significantly longer in the untreated control group as compared to the sham group, whereas the APCtreatment group did not differ from the sham group. Mean $+\mathrm{SD}$ of six experiments. ${ }^{*} P<0.05$ versus sham
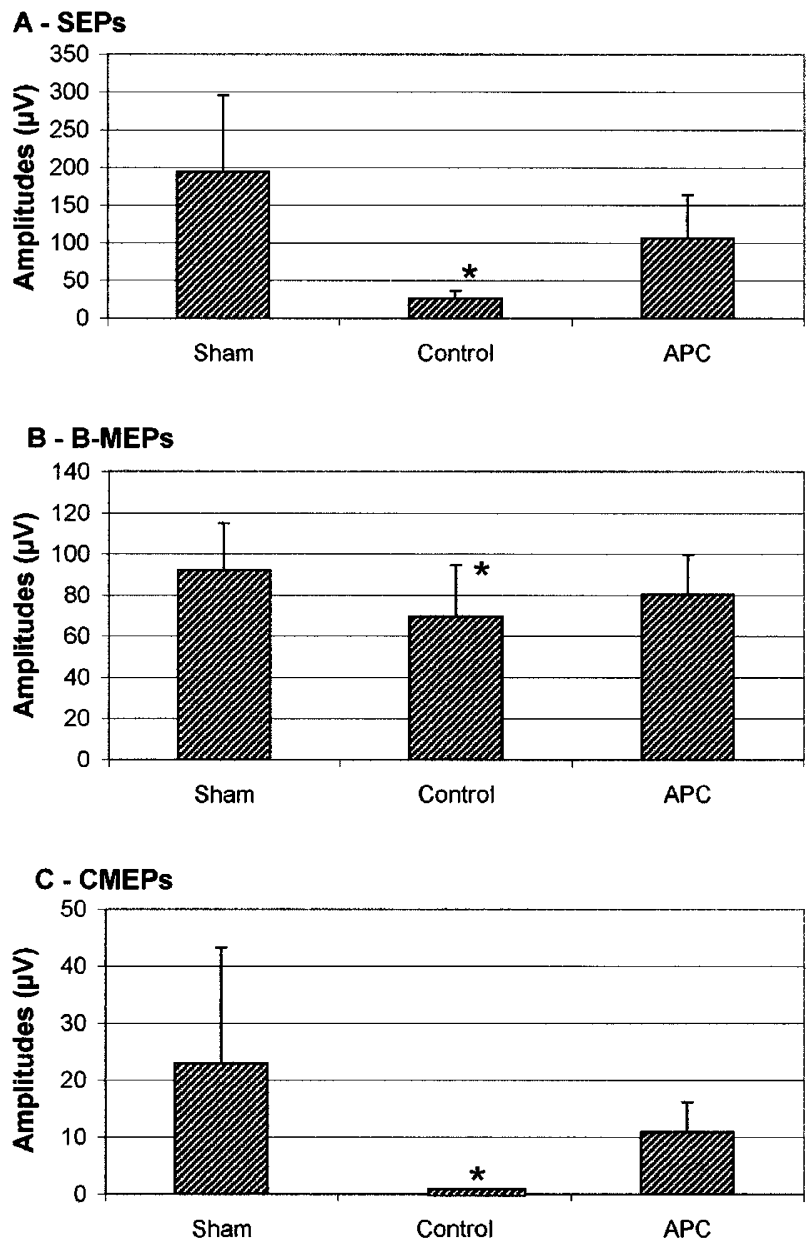

Figure 7 Effect of APC on the amplitude of SEPs (A), BMEPs (B) and CMEPs (C). APC-treatment significantly attenuated the reduction in SEP amplitude (A). B-MEP amplitudes in the untreated control group were significantly lower than in the sham group (B). No CMEPs were recorded in the untreated control group, while they were present in the APC-treatment group (C). Mean \pm SD of six experiments. $* P<0.05$ versus sham and APC-groups (A) and versus sham (B) bances were reduced by APC-treatment for up to 8 weeks following SCI. APC also preserved axonal

A

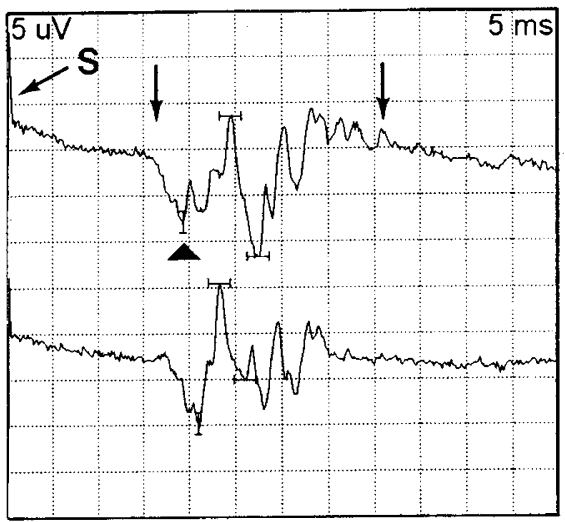

B

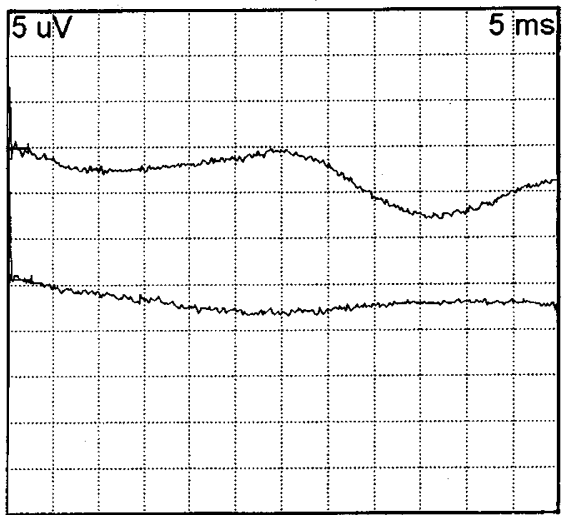

C

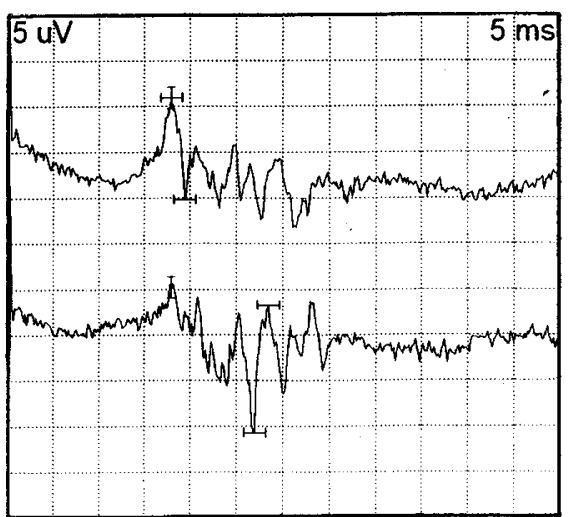

Figure 8 Effect of APC on CMEPs. While no CMEPs were recorded in the untreated control group (B), APC-treated animals had preserved CMEPs $(\mathbf{C})$ that were similar to CMEPs in the sham group (A). Representative recordings are shown. (A) Upper signal: s, stimulus artifact; arrows indicate signal onset and end; the vertical cursor (arrowhead) marks the first peak (latency); the horizontal cursors indicate the maximal amplitude within the signal 
Table 1 Histological parameters

\begin{tabular}{lcc}
\hline & Control & APC \\
\hline Length $(\mathrm{mm})$ & $3.49 \pm 1.7$ & $2.96 \pm 1.2$ \\
Spared WM $(\%)$ & $28.0 \pm 7.73$ & $36.9 \pm 14.13$ \\
Gray lesion area $\left(\mathrm{mm}^{2}\right)$ & $17.63 \pm 5.11$ & $10.93 \pm 6.23$ \\
\hline
\end{tabular}

All three parameters indicated a smaller lesion size in the APC-treatment group compared to the untreated control group. However, these differences did not reach significance. Mean \pm SD of six experiments

integrity as determined by electrophysiological recordings at the end of the survival period. Thus, attenuation of SCI-induced deficits by APC was observed at a time point that was well after the cessation of spontaneous functional recovery, which occurs within the first 4 weeks after SCI. ${ }^{17-19}$ These findings suggest that under conditions of experimental SCI, APC provides neuroprotection that results in a persistent effect on outcome.

Although histological findings indicated a smaller lesion size in the APC-treatment group compared to the untreated control group, we could not find significant differences between these groups. Although functional recovery has been shown to depend on the amount of spared spinal cord tissue, ${ }^{20-23}$ some reports indicate that the link between recovery and spared tissue may not always be clearly visible. Fehlings and Tator ${ }^{20}$ found that at severe to medium degrees of injury, functional outcome improves exponentially with increasing numbers of surviving axons. Similarly, Young and coworkers $^{21,22}$ demonstrated in animal models that motor function can recover to normal levels after SCI if as few as $4 \%$ to $6 \%$ of the cortical motor neurons regain physiologic connection through the injured spinal cord segment to the caudal spinal cord. Thus, even a very small increase in surviving axons could have a great impact on functional outcome while not being detectable by conventional histological means. Moreover, not all descending motor tracts contribute equally to motor function recovery. ${ }^{20}$ Slight inter-animal differences in the relative amounts of surviving axons of different motor tracts may thus have a considerable impact on functional recovery without any measurable histological changes.

In the present study, no CMEPs were recorded in the control group, while they were present in the APCtreatment group. We have previously shown that the CMEPs recorded in this study reflect integrity of the corticospinal tract (CST): transection of the CST completely and irreversibly abolished the signals. ${ }^{16}$ Thus, these signals can be considered to be of true cortical origin. Several studies demonstrated that the persistence after SCI of axons in the CST was strongly associated with residual hind limb locomotor function and have focused on this tract to evaluate the efficacy of various therapeutic strategies. ${ }^{24-26}$ Thus, APC may reduce the compression-induced motor disturbances by preserving the function of the CST.
Results of previous studies indicated that APC's beneficial effect in SCI may be due to its antiinflammatory rather than its anticoagulant property. Taoka et $a l^{10}$ showed that the attenuation of motor disturbances following traumatic SCI was associated with the prevention of neutrophil infiltration of the lesioned spinal cord. Similarly, Murakami et $a l^{27}$ showed that APC prevented lipopolysaccharideinduced pulmonary vascular injury in rats by inhibiting leukocyte activation. In a rat model of liver ischemia and reperfusion, APC significantly decreased neutrophil accumulation and cytokineinduced neutrophil chemoattractant. ${ }^{28}$

Two mechanisms of APC's anti-inflammatory effect have been proposed. On the one hand, APC is thought to inhibit neutrophil recruitment and activation by down-regulating cytokine production by monocytes. ${ }^{9}$ On the other hand, it has also been shown that APC attenuates the response of endothelial cells to inflammatory mediators by a specific receptor. ${ }^{29}$ Several reports indicate that endothelial cell activation plays an important role in SCI. ${ }^{12,30}$ Hamada et $a l^{12}$ showed rapid up-regulation of endothelial cell adhesion molecules with subsequent neutrophil infiltration in injured spinal cord. Thus, APC may protect injured tissue from secondary auto-destruction by attenuating endothelial activation and cytokine release from monocytes.

While APC's anticoagulant activities most likely do not contribute to the beneficial effect of APC in acute $\mathrm{SCI},{ }^{10}$ it may provide additional benefits in a clinical setting. Thromboembolism has been reported to occur in $70 \%$ to $100 \%$ of patients who have complete motor paralysis after SCI and is a major cause of morbidity and mortality in patients with SCI. ${ }^{31-35}$ Thus, APC therapy may be beneficial to patients with SCI not only by improving the motor recovery, but also by preventing thromboembolism through its anticoagulant potency.

In conclusion, the present study showed that pretreatment with APC significantly reduced the motor disturbances induced by SCI not only in acute phase but also in the chronic stage. Since APC is a physiological agent and no side effects have so far been found, ${ }^{36}$ APC seems worthy of further investigations into its potential use as a neuroprotective agent in acute SCI. In particular, the neuroprotective efficacy of APC has to be evaluated in clinically relevant posttreatment time frames.

\section{Acknowledgements}

We gratefully acknowledge the excellent technical assistance of Monika Schuller and Doris Riegelnegg. We thank Prof Hans-Peter Schwartz (Baxter AG, Vienna, Austria) for kindly providing activated protein $\mathrm{C}$, and Prof Kenji Okajima (Medical School, Kumamoto University, Japan) for valuable suggestions. 


\section{References}

1 Stover SL, Fine FR. The epidemiology and economics of spinal cord injury. Paraplegia 1987; 24: 225-228.

2 Bracken MB et al. A randomized, controlled trial of methylprednisolone or naloxone in the treatment of acute spinal-cord injury. New Engl J Med 1990; 322: $1405-1411$.

3 Young W. Secondary CNS injury. J Neurotrauma 1998; 5: 219 221.

4 Zeidman SM, Ling GS, Ducker TB, Ellenbogen RG. Clinical applications of pharmacologic therapies for spinal cord injury. $J$ Spinal Disord 1996; 9: 367-380.

5 Demopoulos HB et al. The fine structure of endothelial structures in the microcirculation of experimentally injured feline spinal cords. Scan Electron Microsc 1978; 2: 677-680.

6 Taoka Y, Okajima K. Spinal cord injury in the rat. Prog Neurobiol 1998; 56: $341-358$

7 Walker FJ, Sexton PW, Esmon CT. The inhibition of blood coagulation by activated protein $\mathrm{C}$ through the selective inactivation of activated factor V. Biochim Biophys Acta 1979; 571: $333-342$.

8 Esmon CT. The protein C anticoagulant pathway. Arterioscler Thromb 1992; 12: $135-145$.

9 Grey S, Hau H, Salem HH, Hancock WW. Selective effects of protein $\mathrm{C}$ on activation of human monocytes by lipopolysaccharide, interferon-g, or PMA: Modulation of effects on CD11b and CD14 but not CD25 or CD54 induction. Transplant Proc 1993; 25: $2913-2914$

10 Taoka $\mathrm{Y}$ et al. Activated protein $\mathrm{C}$ reduces the severity of compression-induced spinal cord injury in rats by inhibiting activation of leukocytes. J Neuroscience 1998; 18: 1393-1398.

11 Gresele $\mathrm{P}$ et al. Activated human protein $\mathrm{C}$ prevents thrombininduced thromboembolism in mice. Evidence that activated protein $\mathrm{c}$ reduces intravascular fibrin accumulation through the inhibition of additional thrombin generation. J Clin Invest 1998; 101: $667-676$.

12 Hamada $\mathrm{Y}$ et al. Involvement of an intracellular adhesion molecule-dependent pathway in the pathogenesis of secondary changes after spinal cord injury in rats. $J$ Neurochem 1996; 66: $1525-1531$.

13 Rivlin AS, Tator $\mathrm{CH}$. Objective clinical assessment of motor function after experimental spinal cord injury in the rat. $J$ Neurosurg 1997; 47: $577-581$.

14 Kunkel-Bagden E, Bregman BS. Methods to assess the development and recovery of locomotor function after spinal cord injury. Exp Neurol 1993; 119: 153-164.

15 Nashmi R, Imamura H, Tator CH, Fehlings MG. Serial recordings of somatosensory and myolectric motor evoked potentials: role in assessing functional recovery after graded spinal cord injury in the rat. $J$ Neurotrauma $1997 ; \mathbf{1 4}$ : $151-159$.

16 Schlag MG, Hopf R, Redl H. Recording of cortically and brainstem evoked muscle potentials in a chronic rat model. Shock (Suppl) 1999; 12: 3.

17 Saruhashi Y, Young W. Effect of mianserin on locomotory function after spinal cord hemisection in rats. Exp Neurol 1994; 129: $207-216$.

18 Little JW, Harris RM, Sohlberg RC. Locomotor recovery following subtotal spinal cord lesions in a rat model. Neurosci Lett 1988; 87: 189 - 194.
19 Behrmann DL, Bresnahan JC, Beattie MS, Shah BR. Spinal cord injury produced by consistent mechanical displacement of the cord in rats. J Neurotrauma 1992; 9: $197-217$.

20 Fehlings MG, Tator $\mathrm{CH}$. The relationship among the severity of spinal cord injury, residual neurological function, axon counts, and counts of retrogradely labeled neurons after experimental spinal cord injury. Exp Neurol 1995; 132: 220-228.

21 Blight AR, Young W. Central axons in injured cat spinal cord recover electrophysiological function following remyelination by Schwann cells. J Neurol Sci 1989; 91: 15-34.

22 Young W. Secondary injury mechanisms in acute spinal cord injury. J Emerg Med 1993; 11: 13-22.

23 Basso DM, Beattie MS, Bresnahan JC. Graded histological and locomotor outcomes after spinal cord contusion using the NYU weight-drop device versus transection. Exp Neurol 1996; 139: $244-256$.

24 Schnell L, Schwab ME. Axonal regeneration in the rat spinal cord produced by an antibody against myelin-associated neurite growth inhibitors. Nature 1990; 343: $269-272$.

25 Cheng H, Cao Y, Olson L. Spinal cord repair in adult paraplegic rats: partial restoration of hind limb function. Science 1996; 273: $510-513$.

26 Grill $\mathrm{R}$ et al. Cellular delivery of neurotrophin-3 promotes corticospinal axonal growth and partial functional recovery after spinal cord injury. J Neuroscience 1997; 17: 5560 - 5572.

27 Murakami $\mathrm{K}$ et al. Activated protein $\mathrm{C}$ attenuates endotoxininduced pulmonary vascular injury by inhibiting activated leukocytes in rats. Blood 1996; 87: 642-647.

28 Yamaguchi $\mathrm{Y}$ et al. Pretreatment with activated protein $\mathrm{C}$ or active human urinary thrombomodulin attenuates the production of cytokine-induced neutrophil chemoattractant following ischemia/reperfusion in rat liver. Hepatology 1997; 25: 11361140.

29 Taylor FB et al. The endothelial cell protein $\mathrm{C}$ receptor aids in host defence against escherichia coli sepsis. Blood 2000; 95: $1680-1686$.

30 Tonai $\mathrm{T}$ et al. Possible involvement of interleukin-1 in cyclooxygenase- 2 induction after spinal cord injury in rats. $J$ Neurochem 1999; 72: 302-309.

31 Silver JR. Prophylactic use of anticoagulant therapy in prevention of pulmonary emboli in one hundred consecutive spinal injury patients. Paraplegia 1974; 12: $188-196$

32 Frisbie JH, Sharma RK. Circadian rhythm of pulmonary embolism in patients with acute spinal cord injury. Am J Cardiol 1992; 70: $847-848$.

33 Todd JW, Frisbie JH, Rossier AB. Deep vein thrombosis in acute spinal injury: a comparison of ${ }^{125}$ I-fibrinogen scanning, impedance plethysmography, and venography. Paraplegia 1976; 14: $50-57$

34 Rossi EC, Green D, Rosen JS. Sequential change in factor VIII and platelets preceding deep vein thrombosis in patients with spinal cord injury. Br J Haematol 1980; 45: 143 - 151 .

35 Myllynen P, Kammonen M, Rokkanen P. Deep venous thrombosis and pulmonary embolism in patients with acute spinal cord injury: a comparison with non-paralyzed patients immobilized due to spinal fracture. J Trauma 1985; 25: 541 - 546.

36 Okajima $\mathrm{K}$ et al. Treatment of patients with disseminated intravascular coagulation by protein C. Am J Hematol 1990; 33: $277-278$ 